\title{
Herbicide resistance endowed by enhanced rates of herbicide metabolism in wild oat (Avena spp.)
}

\begin{abstract}
The biochemical basis of resistance to the acetyl-coenzyme A carboxylase (ACCase)inhibiting herbicide diclofop-methyl was investigated in a resistant wild oat population (R1), which does not exhibit a resistant ACCase. Rates of foliar uptake and translocation of [14C]diclofop were the same in the R1 vs. susceptible (S) populations. However, the level of phytotoxic diclofop acid was always found to be lower in the R1 vs. S plants, with a concomitant higher level (up to 1.7-fold) of nontoxic polar diclofop metabolites in R1 relative to the $\mathrm{S}$ plants. These results indicate that a noni target-site-based mechanism of enhanced rate of diclofop acid metabolism confers resistance in population R1. Moreover, the highperformance liquid chromotography elution profile of the major diclofop metabolites in R1 is similar to that of wheat, suggesting resistance in individuals of population R1 involves a wheat-like detoxification system mediated by cytochrome P450 monooxygenases. In addition, lower level of tissue diclofop acid was also observed using nonradioactive ultraperformance liquid chromatographyï mass spectrometry analysis in resistant individuals of three other resistant wild oat populations (R2, R3, and R4) known to posses ACCase gene resistance mutations. These results establish that either one or at least two independent resistance mechanisms (target-site ACCase resistance mutations and nonï target-site enhanced rates of herbicide metabolism) can be present in individual wild oat plants.
\end{abstract}

Keyword: Herbicide uptake and translocation; Multiple resistance mechanisms; Non-targetsite based resistance 